\title{
Les enjeux philosophiques et sociologiques d'une redéfinition actionniste de la « construction sociale » des sciences
}

Michel Dubois

\section{(2) OpenEdition \\ Journals}

Édition électronique

URL : http://journals.openedition.org/ress/585

DOI : $10.4000 /$ ress.585

ISSN : 1663-4446

Éditeur

Librairie Droz

\section{Édition imprimée}

Date de publication : 1 août 2002

Pagination : 185-198

ISBN : 2-600-00806-3

ISSN : 0048-8046

Référence électronique

Michel Dubois, « Les enjeux philosophiques et sociologiques d'une redéfinition actionniste de la " construction sociale » des sciences », Revue européenne des sciences sociales [En ligne], XL-124 | 2002, mis en ligne le 01 décembre 2009, consulté le 02 mai 2019. URL : http:// journals.openedition.org/ress/585; DOI : 10.4000/ress.585 


\section{LES ENJEUX PHILOSOPHIQUES ET SOCIOLOGIQUES D'UNE REDÉFINITION ACTIONNISTE DE LA «CONSTRUCTION SOCIALE» DES SCIENCES}

L'identité de la sociologie des sciences peut être appréhendée de diverses manières. L'une d'elles consiste à rechercher la nature et l'origine de cette identité par une analyse interne de ses dimensions intellectuelles et institutionnelles. Cette analyse privilégiera d'une part l'observation de l'évolution des théories et méthodes sociologiques appliquées aux sciences: le renouveau d'intérêt pour l'héritage classique de la sociologie de la connaissance, la critique du fonctionnalisme prêtée à l'approche mertonienne des institutions et professions scientifiques, la valeur démonstrative accordée aux études monographiques et à leur accumulation, etc. Elle prendra en compte, d'autre part, les facteurs généraux liés directement ou indirectement aux divers aspects de cette évolution: le renouvellement des générations de chercheurs et l'intensification des relations de coopération et concurrence entre les groupes de recherche, la mise en place des programmes de financement, la création de structures associatives nationales et internationales, la multiplication de revues spécialisées, etc.

Cette démarche est indéniablement utile: elle met en évidence la pluralité des facteurs associée à la dynamique interne de la sociologie des sciences. Elle donne à comprendre la reconnaissance académique d'un style de recherche particulier ${ }^{1}$. Elle tend cependant de façon compréhensible à en surévaluer le caractère à la fois unifié et autonome. L'analyse sociologique des sciences n'est pas un espace intellectuel et institutionnel hermétique aux frontières clairement délimitées. Elle s'est certes construite à partir de problématiques, de répertoires conceptuels et thématiques élaborés par la tradition sociologique - on pense bien sûr aux éléments forgés par E. Durkheim, M. Scheler, K. Mannheim, P. Sorokin pour s'en tenir aux classiques $^{2}-$, mais cette construction passe tout autant, parfois même davantage

1 On trouve un bel exemple de cet exercice pour la tradition mertonienne chez J. Cole, H. Zuckerman, The emergence of a scientific speciality: the self exemplifying case of the sociology of science, in Coser L. (ed), The idea of social structure, New York, Harcourt Brace Janovich, 1975. Voir également G. Lemaine., La sociologie de la science en France, in «La recherche en sciences humaines », Paris, Ed. du CNRS, 1979. Et plus récemment M. Dubois, La nouvelle sociologie des sciences, Paris, Presses Universitaires de France, 2001, tout particulièrement le chap. 1.

2 E. Durkheim, Les formes élémentaires de la vie religieuse, Paris, Presses Universitaires de France, 1979 (1912); M. Scheler, Les formes de la connaissance et la société, Leipzig, Der Neue-Geist Verlag, (1926), traduction française partielle: Problèmes de sociologie de la connaissance, Presses Universitaires de France, Paris, 1993; Mannheim K., Ideology and utopia; an introduction to the sociology of knowledge, Brace and Company, New York, 1936 (1929), traduction française partielle: Idéologie et utopie, Librairie Marcel Rivière, Paris, 1952; P. Sorokin, Social and Cultural dynamics, Fluctuation of systems of Truth, Ethics, and Law, vol. II, American Book company, 1937. 
dans certains cas, par des échanges et des transferts avec des traditions et perspectives non sociologiques. Nombre de commentateurs n'hésitent pas à voir dans l'intégration de la sociologie des sciences dans le courant plus général des «études sociales de la science» la conséquence naturelle de l'importance de ces transferts et de ces échanges. On peut discuter des risques que fait peser sur la sociologie une telle intégration, notamment du point de vue de sa dilution dans un questionnement social et de son instrumentalisation à des fins idéologiques ${ }^{3}$. Il n'en demeure pas moins vrai que son identité disciplinaire apparaît dès lors comme influencée par la manière dont ses acteurs se représentent les similitudes et différences de leur démarche au regard de celles des non-sociologues avec lesquels ils partagent un intérêt général pour l'étude des sciences. L'identité sociologique acquiert une dimension relationnelle et représentationnelle.

\section{A. MODALITÉS ET ENJEU DU DIALOGUE ENTRE PHILOSOPHES ET SOCIOLOGUES DES SCIENCES}

Parmi les dialogues interdisciplinaires ayant contribué à façonner l'identité de la sociologie des sciences, celui entrepris avec les philosophes occupe une place particulière. Les sociologues des sciences se sont certes intéressés aux travaux des anthropologues, des ethnométhodologues, des historiens, des économistes. J'ai essayé ailleurs d'établir les raisons de leur fascination à l'égard des premiers ${ }^{4}$. Mais jamais ces discussions n'ont connu une ampleur et une intensité comparable à celle nouée avec les philosophes. Un observateur pressé ${ }^{5}$ pourrait avoir de «bonnes raisons» de croire que la sociologie des sciences reste une «province» turbulente de la philosophie tant certains de ses représentants semblent davantage préoccupés par des problèmes de théorie de la connaissance-comme ceux du réalisme, de l'idéalisme, du relativisme, du rationalisme - que par la compréhension et l'explication des divers aspects sociaux de la pratique scientifique. Et ce sentiment, me semble-t-il, risquera de paraître d'autant plus fondé s'il repose sur l'indifférenciation postulée de la sociologie des sciences et des «études sociales de la science».

\section{L'intégration-adaptation, la critique méthodique, le débat immobile ou la stratégie de «l'homme de paille»}

Mon but n'est pas de restituer de façon exhaustive ce dialogue entre philosophes et sociologues autour de l'analyse des sciences. Je propose d'isoler trois de ses modalités récurrentes - l'intégration-adaptation, la critique méthodique, le

\footnotetext{
Cf. M. Dubois, L'affaire Sokal: cultural studies et sociologie relativiste des sciences. in «Revue française de sociologie», XXXIX-3, 1998.

4 Cf. M. Dubois, 2001, op. cit., chap.5.

5 Pressé, c'est-à-dire qui tenterait de se donner une représentation d'ensemble du domaine à partir de la lecture d'un ensemble limité de recueils ou de manuels, tels que M. Hollis, S. Lukes (eds.), Rationality and relativism, Oxford, Basil Blackwell, 1982; A. Chalmers, La fabrication de la science, Ed. de la découverte, 1991; R. Boudon \& M. Clavelin (eds.), Le relativisme est-il résistible? - Regards sur la sociologie des sciences, Paris, Presses Universitaires de France, 1994.
} 
débat immobile ou stratégie de «l'homme de paille»- et de définir ce qui me parait être l'un de ses principaux enjeux.

Une première façon de penser le rapport entre philosophie et sociologie des sciences passe par l'étude des transferts théoriques et conceptuels d'un domaine à un autre et plus encore l'adaptation requise par ces transferts. Il est largement admis que la réorientation de l'analyse sociologique vers la dimension cognitive des sciences tient à la manière dont une génération entière de sociologues s'est réapproprié des théories, catégories ou concepts philosophiques ${ }^{6}$. Le cas le plus célèbre est bien sûr celui du concept de «paradigme». Les sociologues ont rapidement perçu l'utilité de ce concept kuhnien. Mais ils ont tout aussi rapidement divergé dans leur manière de concevoir cette utilité. Certains, restés proche de la tradition mertonienne, établissent une relation de synonymie entre «paradigme» et «structure sociale interne » ${ }^{7}$. D'autres, se consacrant à l'étude de l'émergence des spécialités scientifiques, pensent ce concept comme un principe d'interdépendance entre facteurs sociaux et facteurs cognitifs ${ }^{8}$. D'autres enfin, réunis un temps autour au «programme fort» de D. Bloor et B. Barnes, interprètent ce même concept comme l'expression du caractère fondamentalement culturel de l'activité scientifique et partant la légitimation d'une approche strictement culturaliste des sciences'. Le transfert d'un concept d'un domaine à un autre - ici de la philosophie vers la sociologie — , et plus encore le travail d'adaptation-interprétation qui s'y rapporte, opère comme un puissant principe de démarcation interne ${ }^{10}$.

Les concepts de «forme de vie», «jeux de langage» ou «air de famille» développés par Wittgenstein constituent un autre exemple classique des divers aspects de ce processus. Ces concepts ont rencontré un succès considérable chez les sociologues des sciences suite à un recadrage théorique initié par $\mathrm{P}$. Winch ${ }^{11}$. Certains ont même proposé de voir en Wittgenstein l'origine indirecte de l'émergence d'une socio-épistémologie ${ }^{12}$. Mais là encore, comme pour le concept kuhnien de

6 Pour des éléments d'explication de cette réorientation, voir J. Ben-David, Scientific growth, University of California Press, 1991, particulièrement l'introduction de G. Freudenthal. Cf. également M. Dubois, Introduction à la sociologie des sciences, Paris, Presses Universitaires de France, 1999.

7 Cf. D. Crane, La nature de la communication et des influences dans le domaine scientifique in «Revue Internationale des Sciences sociales», XXII, 1, 1970; S. Cole, J. Cole, Social stratification in science, Chicago, Chicago University Press, 1973; J. Cole, H. Zuckerman, 1975, op.cit

8 Cf. M. Mulkay, Some suggestions for sociological research in «Science studies», 1, 1971; G. Lemaine, R. MacLeod, M. Mulkay, P. Weingart (eds), Perspectives on the emergence of scientific disciplines, The Hague and Paris, Mouton, 1976.

9 Cf. B. Barnes, T. S.Kuhn and the social sciences, The Macmillan Press, 1982; et également l'introduction M. Callon et B. Latour à M. Callon, B. Latour (eds), La science telle qu' elle se fait, Paris, Ed. de la découverte, 1991.

10 T. Pinch propose une relecture dualiste - conservateurs vs radicaux - du transfert de la théorie kuhnienne vers la sociologie des sciences, cf. P. Pinch, Kuhn - the conservative and radical interpretations : are some mertonians «kuhnians»and some kuhnians 'mertonians'? in « Social Studies of Science», vol. 27, 1997 (1982).

11 P. Winch, The idea of social science, Londres, Routledge \& Kegan Paul, 1958

12 D. Phillips, Wittgenstein and scientific knowledge : a sociological perspective, Londres, Macmillan, 1977; D. Bloor, Wittgenstein : a social theory of knowledge, New York, Columbia University Press, 1983; W. Sharrock, R. Anderson, The wittgenstein connection in «Human studies», 7, 1984; H. Collins, Changing order ; replication and induction in scientific practice, Londres, Sage, 1985. 
paradigme, les sociologues s'opposent dans leur façon d'adapter ces concepts à leurs objets spécifiques. Ces lignes de fractures sont particulièrement sensibles dans le volume collectif édité par A. Pickering en 1992: «Science as practice and culture ». D. Bloor y pose une distinction rigide, à l'intérieur du domaine de l'analyse sociologique des sciences, entre les «wittgensteiniens de gauche» et les «wittgensteiniens de droite». Les premiers exploitant selon lui pleinement la dimension sociologique latente des concepts wittgensteiniens - celle du moins que $\mathrm{D}$. Bloor prête à Wittgenstein et qui s'apparente à un conventionnalisme radi$\mathrm{cal}^{13}$. Les seconds les réduisant à ses yeux à une forme d'internalisme enracinée dans une théorie de l'action et du sens jugée tout à la fois «improbable, restreinte, et empiriquement inadéquate ${ }^{14}$. Ce à quoi M. Lynch répond que les wittgensteiniens de gauche représentés par D. Bloor passent à côté de ce qui fait l'essentiel de l'apport de Wittgenstein à l'analyse des pratiques scientifiques ${ }^{15}$.

Une seconde forme générale d'interaction prend la forme non pas d'un transfert conceptuel ou théorique mais d'un dialogue critique autour d'objets ou de thèmes délimités - dialogue fondé sur l'acceptation par les parties discutantes d'un certain nombre d'éléments définitionnels de base. Un premier exemple classique de ce type d'échange est le débat organisé à Toronto en 1980 entre D. Bloor et L. Laudan sur la pertinence du principe de symétrie; débat dont on trouve la trace dans les volumes 11 et 12 de "Philosophy of the social sciences ${ }^{16}$. L. Laudan pose le problème de la scientificité de l'explication sociologique de la connaissance scientifique revendiquée par D. Bloor. «Là où les philosophes, souligne-t-il avec ironie, désespèrent d'établir un jour une démarcation nette entre le scientifique et le non scientifique [...] Bloor non seulement affirme qu'une telle démarcation existe mais qu'il l'a trouvée». Sur les quatre principes auxquels D. Bloor identifie cette scientificité - causalité, impartialité, réflexivité, symétrie -, L. Laudan accorde une attention particulière à l'idée selon laquelle le sociologue n'aurait pas à changer de registre explicatif en fonction de la nature du phénomène qu'il étudie. Celle-ci lui paraît difficilement acceptable. Son argument se déploie en deux temps. Il consiste tout d'abord à montrer que D. Bloor confond sous ce principe général trois situations pourtant bien distinctes: la symétrie épistémique (même type d'explication pour croyances vraies et fausses), la symétrie rationnelle (même type d'explication pour croyances rationnelles et irrationnelles), la symétrie pragmatique (même type d'explication pour les croyances efficaces et inefficaces). Et que la mise en œuvre d'un registre explicatif unique pour chacune de ces situations peut avoir pour conséquence l'occultation pure et simple de la capacité des acteurs scientifiques à «raisonner». Il consiste ensuite à montrer que D. Bloor ne définit à aucun moment précisément la nature de ce registre explicatif. «Dire qu'il faut expliquer les croyances par le 'même type de cause', écrit

\footnotetext{
13 Sur la nature de ce conventionnalisme, cf. M. Dubois (2001), op. cit., chap. 4, section D.

14 D. Bloor, Left and right wittgensteinians in A. Pickering ( ed.), Science as practice and culture, The University of Chicago Press, Chicago, 1992.

15 M. Lynch, Extending Wittgenstein: the pivotal move from epistemology to the sociology of science, in Pickering A. (ed), op. cit., 1992.

16 L. Laudan, The pseudo-science of science? in «Philosophy of the social sciences », vol. 11, 1981;

D. Bloor, The strengths of the strong programme in «Philosophy of the social sciences », vol. 11, 1981 ; L. Laudan, More on Bloor in «Philosophy of the social sciences », vol. 12, 1982
} 
L. Laudan, nécessite au préalable la spécification d'une taxinomie des types de causes. Tant que l'on ne sait pas comment classer les causes, il nous est impossible de répondre véritablement à l'injonction d'utiliser la même sorte de causes». D. Bloor, dans sa réponse à L. Laudan, a beau essayer de faire valoir les «forces » du programme fort à partir d'une distinction entre cause rationnelle et cause irrationnelle d'une part, rationalité naturelle et rationalité normative d'autre part, jamais il ne semble en mesure d'apporter de réponses convaincantes aux difficultés identifiées par son contradicteur.

Un autre exemple, plus récent, de l'interaction entre philosophie et sociologie des sciences peut être recherché dans l'échange entre A. Franklin et H. Collins autour du problème de la régression de l'expérimentateur ${ }^{17}$. Il faut souligner, à la suite de Y. Gingras et B. Godin ${ }^{18}$, que ce débat réactualise une thématique classique de la tradition philosophique depuis le scepticisme grec jusqu'à l' "Apologie de Raymond Sebond» de Montaigne. Pour H. Collins, comme pour B. Latour sous d'autres formes, tout travail expérimental dès lors qu'il sert de principe de vérification est confronté à un cercle vicieux : pour vérifier l'existence d'un fait il faut un dispositif expérimental, mais pour savoir si ce dispositif fonctionne correctement il faut qu'il soit capable de produire le fait recherché. Dans la célèbre étude de cas qu'il consacre à la détection des ondes gravitationnelles par J. Weber ${ }^{19}$, H. Collins se fixe un double objectif: 1- démontrer la réalité de cette circularité à partir de l'étude de la controverse née du dispositif expérimental imaginé par J. Weber, 2- démontrer l'influence de facteurs extrascientifiques dans la rupture d'une situation caractérisée par son indétermination logique. A. Franklin reprend l'étude de cas proposée par $\mathrm{H}$. Collins mais affirme que la régression de l'expérimentateur - régression promue par H. Collins au rang de condition de possibilité de l'analyse sociologique - est tout simplement étrangère au rejet des résultats de J. Weber par la communauté scientifique. La reconstitution des arguments échangés par J. Weber et ses contradicteurs montre certes que les scientifiques, dans certaines situations, n'appliquent pas une logique formelle unifiée et contraignante pour évaluer la portée d'un travail expérimental. Mais cela ne suffit nullement à prouver qu'ils agissent sur fond d'indétermination radicale et donc indépendamment de tout critère méthodologique et épistémologique. Le travail de A. Franklin consiste précisément à reconstituer progressivement l'accumulation des conditions de crédibilité des arguments avancés par ceux qui s'opposent à J. Weber: les procédures de vérification croisée, d'échange de données et de programmes d'analyse, de recalibrage des dispositifs expérimentaux, etc. Et A. Franklin de conclure sur la base de ce matériau empirique que la communauté scientifique avait des raisons «fortes » de rejeter les résultats de J. Weber et d'ac-

17 H. Collins, A strong confirmation of the experimenters' regret in «Studies in History and Philosophy of Modern Physics», 25, 1994; A. Franklin, How to avoid the experimenters' regret, in «Studies in History and Philosophy of Modern Physics», 25, 1994.

18 Y. Gingras, B. Godin, The experimenter's regress : from skepticism to argumentation, in «Cahiers d'épistémologie », n 210, Publications UQAM, 2000.

19 H. Collins, Les sept sexes : étude sociologique de la détection des ondes gravitationnelles, dans Callon M., Latour B. eds., op. cit., 1991. 
cepter ceux de ses critiques ${ }^{20}$. «Même si aucune règle formelle n'a été appliquée [dans le règlement de cette controverse], écrit-il, la procédure a clairement suivi les normes générales de l'expérimentation scientifique».

Une troisième modalité du dialogue entre philosophes et sociologues des sciences, plus spectaculaire mais indéniablement moins riche que la précédente, est celle de la polémique, de l'invective et plus globalement de tout ce qui peut prendre la forme d'un «débat immobile ${ }^{21}$. On est ici dans la situation du dialogue de sourds où les partis en présence donnent l'illusion de l'échange pour mieux écarter la possibilité d'une critique rationnelle. Une stratégie argumentative fréquemment observable dans cette situation est celle dite de l' «homme de paille». Elle consiste à faire valoir son point de vue en construisant de toutes pièces un opposant auquel on prête des positions suffisamment caricaturales pour s'en débarrasser d'autant plus facilement. Les sociologues des sciences postkuhniens ont fait un usage intensif de cette pratique vis-à-vis tant des sociologues mertoniens que des philosophes des sciences. Ils ont pendant très longtemps fait de l'opposition à la «représentation standard de la connaissance» un vecteur d'identité collective tout en se gardant bien de s'interroger sur la réalité effective de cette représentation attribuée aux philosophes. Et c'est à bon droit que ces derniers ont régulièrement fait observer que l'image conventionnelle de la science contre laquelle prétendaient se battrent les sociologues des sciences n'avait en dernière analyse de réalité qu'argumentative. Lors de la polémique internationale qui a suivi l'affaire dite Sokal ${ }^{22}$, la même stratégie a été utilisée cette fois par les philosophes, accompagnés il est vrai de quelques représentants des sciences de la nature, contre les sociologues des sciences. Ils ont produit l'image d'une sociologie des sciences uniformément livrée à un relativisme radical pour lequel les vérités scientifiques seraient le produit d'activités sociales et linguistiques contingentes; et ce faisant, c'est-à-dire en ignorant plus ou moins délibérément la diversité interne à l'analyse sociologique des sciences, ils se sont facilité l'argumentation en faveur d'une approche non sociologique des sciences. Conscients du danger, les sociologues ont régulièrement tenté de se démarquer, avec un succès mitigé, de ce qu'ils ont présenté comme un discours «ultra minoritaire » cantonné aux «variantes fanatisées » des cultural studies, ou encore comme des «excès de jeunesse » de la part de sociologues considérablement assagis depuis ${ }^{23}$.

\section{Délimitation et complémentarité des approches}

Ce bref rappel de quelques-unes des modalités du dialogue philosophicosociologique autour de l'étude des sciences conduit à l'un de ses principaux enjeux: la délimitation des domaines d'études propres à chaque discipline et plus

20 Il faut souligner que le travail «philosophique» de A. Franklin retrouve les conclusions de l'analyse critique proposée par le sociologue G. Darmon, The asymetry of symetry, in « Social science information », 25, 1986 .

21 Nous reprenons ici le titre de l'ouvrage de M. Doury, Le débat immobile. L'argumentation dans le débat médiatique sur les parasciences, Paris, Kimé, 1997.

22 M. Dubois, op. cit., 1998; M. Dubois, op. cit., 2001, chap. 7.

23 On trouve ce type d'argumentaire dans l'ouvrage collectif dirigé par B. Jurdant (ed), Impostures scientifiques. Les malentendus de l'affaire Sokal, Paris, La découverte, 1998. 
encore la redéfinition de la relation entre les représentations produites par les uns et par les autres. Le renouveau de la sociologie des sciences a été pour beaucoup synonyme d'une modification des frontières établies entre philosophie et sociologie ayant pour conséquence une intégration dans le registre sociologique de questions et de problèmes traités jusqu'alors par les seuls philosophes. On peut avec de bonnes raisons contester ce point de vue en montrant, à partir d'une relecture de la tradition sociologique, que l'étude des dimensions sociales du processus de recherche scientifique est très largement antérieure à l'importation des concepts kuhniens vers la sociologie. La nouveauté n'est pas le déplacement de frontière proprement dit mais la manière dont il a été associé par certains sociologues à une conception «exclusiviste » des points de vue propres à chaque discipline. Ce point - mis en évidence entre autres par R. Boudon dans son analyse des deux sociologies de la connaissance scientifique ${ }^{24}$ - constitue le véritable point d'ancrage des dialogues précédemment évoqués. Si A. Franklin insiste tant sur les raisons qui poussent les scientifiques à rejeter les résultats de J. Weber, c'est parce qu'il a le sentiment que l'explication sociologique proposée par H. Collins suppose l'éviction pure et simple du point de vue épistémologique. De même si L. Laudan discute de façon aussi méthodique les registres dans lesquels s'exercent le principe bloorien de symétrie comme le flou qui entoure sa conception du registre causal de l'explication sociologique, c'est parce qu'il perçoit la démarche du programme fort comme cherchant non pas à compléter mais à se substituer au point de vue philosophique. Et tous deux ont de ce point de vue parfaitement raison. Le causalisme symétrique du programme fort repose bel et bien sur l'idée selon laquelle les structures explicatives de la philosophie et de la sociologie sont «mutuellement exclusives ». Sa radicalité repose tout entière sur cette clause qui implique qu'à un phénomène unique ne peut correspondre simultanément une justification rationnelle et un enracinement social. La force et l'intérêt du dialogue entre philosophes et sociologues dans un premier temps, entre les sociologues dans un second temps ${ }^{25}$, est d'avoir non seulement mis au jour l'absurdité d'une telle croyance mais également et surtout d'avoir généré une réflexion collective sur la nécessaire réorientation de l'explication sociologique des sciences.

\section{B. QUELLE RÉORIENTATION POUR L'ANALYSE SOCIOLOGIQUE DES SCIENCES?}

Quelles sont précisément les modalités de cette réorientation? Je voudrais ici brièvement analyser deux voies à travers lesquelles les sociologues sont fréquemment invités à repenser les termes de leur démarche et par conséquent leur rapport à la philosophie des sciences. J'essaierai de montrer les raisons pour lesquelles aucune d'elles n'est réellement acceptable. Ce qui m'amènera dans la section suivante à la caractérisation d'un programme de recherche d'inspiration actionniste.

24 R. Boudon, Les deux sociologies de la connaissance scientifique, in Boudon R., Clavelin M. (eds), op. cit., 1994.

25 Pour une présentation de ces débats, cf. M. Dubois, op. cit., 1999, chap.1; O. Martin, Sociologie des sciences, Paris, Nathan, 2000. 


\section{Le retrait}

La première réorientation consiste en une réduction du domaine d'investigation de la sociologie des sciences. Cette réduction prend principalement deux formes:

- soit l'abandon pur et simple de l'étude du processus de recherche pour celle, strictement externaliste, de contextes de ce processus. C'est la thèse que défend par exemple G. G. Granger dans son article «Une philosophie des sciences non sociologique est-elle possible ?» ${ }^{26}$. Pour G. G. Granger si le philosophe doit s'efforcer de "proposer une interprétation de la production des œuvres et des concepts scientifiques, c'est-à-dire de donner un sens à ces productions de l'esprit humain», le sociologue doit se limiter aux questions suivantes: "A quelles conditions se forment dans une société une communauté scientifique? Quels sont les types de statut social du savant? Quels sont les rapports des organisations et des organisations scientifiques». Et G. G. Granger de souligner «non pas des concepts techniques aux concepts scientifiques qui concernent le philosophe $»$.

- soit le maintien de l'analyse sociologique des contenus constitutifs du processus de recherche mais pour les seuls contenus auxquels il est impossible d'associer une justification rationnelle. C'est un retour au principe de l'a-rationalité initié par K. Mannheim et c'est pour L. Laudan la seule voie solide sur laquelle la sociologie des sciences peut prétendre s'établir. Pour L. Laudan, «il n'y a pas à douter de la possibilité d'une sociologie de la connaissance pour autant qu'elle travaille dans le cadre de l'hypothèse de l'a-rationalité. Au contraire, c'est un vaste domaine qui est dévolu à la recherche en sociologie cognitive. Quand, par exemple, un savant accepte une tradition de recherche qui est moins adéquate qu'une rivale; quand il poursuit une théorie non progressiste; quand il donne à un problème ou une anomalie plus ou moins de poids qu'ils ne le méritent cognitivement [...] dans tous ces cas nous devons nous tourner vers les sociologues pour comprendre, puisqu'il n'y a aucune possibilité pour une analyse rationnelle de l'action en question ${ }^{27}$.

Pourquoi ces deux voies proposées par les philosophes à l'intention des sociologues des sciences sont-elles inacceptables? Parce que toutes deux - de façon radicale chez G. G. Granger, de façon plus tempérée chez L. Laudan -, non seulement donnent une vision réductrice de l'approche sociologique mais également et surtout reconduisent l'origine même des échecs du programme fort: l'idée selon laquelle «explication rationnelle» d'une part et «explication sociale» sont mutuellement exclusives. Pourquoi les sociologues doivent-ils privilégier l'étude des contextes de la science? Parce que, dit en substance G. G. Granger, les sociologues seraient par principe incapables de donner une vision compréhensive de l'activité scientifique, autrement dit de restituer la dimension significative inhé-

26 G. G. Granger, Une philosophie des sciences non sociologique est-elle possible?, in R. Boudon, M. Clavelin (eds), op. cit., 1994.

27 L. Laudan, La dynamique de la science, Bruxelles, Mardaga, 1987, p.225. 
rente à cette activité. Pourquoi les sociologues doivent-ils s'en tenir à l'étude des «échecs» de l'activité scientifique? Parce qu'affirme L. Laudan les sociologues ont par principe une approche causale de l'explication scientifique et sont donc désarmés dès lors qu'une théorie de la rationalité est indispensable. Mais d'où viennent ces certitudes philosophiques à propos de la nature de la démarche sociologique? D'une confusion compréhensible entre la partie et le tout. L'approche du programme fort, qui constitue la «toile de fond » à partir de laquelle raisonnent ces philosophes, est indéniablement de nature causaliste et occulte la rationalité à l'œuvre dans la pratique scientifique. Mais faut-il voir dans cette approche le dernier mot de la sociologie des sciences, considérer cette exogénéité et cette absence de théorie de la rationalité comme des éléments inhérents à tout programme de recherche sociologique? Rien dans la théorie ou la méthodologie sociologique ne permet d'affirmer une telle chose. Le cadre a priori à partir duquel G. G. Granger comme L. Laudan invitent les sociologues à un retrait total ou partiel de l'étude du processus de la recherche scientifique - et ce faisant établir leur complémentarité avec la philosophie - apparaît comme trop limité.

\section{La dérive}

La seconde voie, tout aussi inacceptable que la précédente, s'apparente à une fuite en avant au cours de laquelle l'analyse sociologique, tentant d'échapper au réductionnisme du programme fort, se transforme en narration pseudo philosophique. Quelles sont les règles de cette narration? Il s'agit d'abandonner l'idée d'une séparation nette entre la «société» et la «nature». Lorsque le sociologue entre dans un laboratoire, affirment les tenants de la théorie de l'acteur-réseau, il lui serait impossible de discerner réellement ce qui est social de ce qui est naturel. Il lui faut donc partir de la «coproduction» de la société et de la nature comme d'un fait primitif pour décrire la manière dont cet ensemble à la fois collectif et coordonné agit et se transforme. Tout objet présent dans un laboratoire constituerait le «porte-parole» du réseau auquel est associée sa matérialisation: son sens comme son application ne peuvent être conçus indépendamment de celui du réseau auquel il appartient. Et ce réseau qui lui donne sens passe tout autant par les humains - un chercheur, un groupe de chercheurs, une organisation - que par les «non-humains »- un dispositif technique, la nature.

Pourquoi cette perspective définie par la théorie l'acteur-réseau ne peut-elle être considérée comme une solution crédible pour réorienter l'analyse sociologique des sciences? Il y a au moins deux raisons à cela. La première est que l'hétérogénéité à laquelle se réfère les représentants de cette théorie ne justifie en rien la thèse de l' «in dissociabilité » ou de la «coproduction» de la société et de la nature. Si les facteurs naturels, sociaux et cognitifs interagissent dans la détermination des comportements scientifiques, cela ne suffit nullement à prouver l'égale intensité de leur influence réciproque et encore moins la nécessité logique de ne pas les séparer analytiquement. Comme l'a bien souligné Y. Gingras ${ }^{28}$, outre le paradoxe qui consiste à établir

28 Y. Gingras, Following scientists through society? Yes, but at arm's length!, Séminaire centre de recherche en histoire des sciences et des techniques, La villette, 1993; traduction française partielle, Un air de radicalisme. Sur quelques tendances récentes en sociologie de la science et de la technologie in «Actes de la recherche en sciences sociales», 108, 1995. 
une relation de synonymie entre «hétérogénéité» et «indissociabilité», les tenants de la théorie de l'acteur-réseau confondent ici «identité » et «relation».

Le second problème est celui des implications de cette indifférenciation des variables sur la capacité du discours sociologique à expliquer quelque chose. Le programme fort postulait dans bien des cas le caractère indéterminé de la nature son incapacité à influer de façon décisive sur le choix des scientifiques - et utilisait cette indétermination pour justifier le principe de l'explication sociologique des sciences. Les philosophes des sciences invitant les sociologues à revenir à l'étude des contextes des sciences ou à se limiter aux croyances irrationnelles ne font au final que reconduire cet usage sociologique du principe d'indétermination comme condition de possibilité de l'explication. Les représentants de la théorie de l'acteur-réseau soulignent à juste titre que cet usage de l'indétermination est dans bien des cas une facilité critiquable mais ils contribuent paradoxalement à introduire une forme d'indétermination plus radicale encore que la précédente: celle de l'acteur. «Le point le plus important, écrit M. Callon, c'est que la théorie de l'acteur réseau n'a aucune théorie stable de l'acteur; [ce qui permet] d'ouvrir les sciences sociales aux non-humains $»^{29}$. Mais y a-t-il un seulement un sens à mettre sur le même plan humains et non-humains, à considérer les microbes comme des «acteurs sociaux $»^{30}$ et des dispositifs techniques comme des quasi-objets capables de «réflexion » ${ }^{31}$ ? Peut-on réellement affirmer que tous les éléments d'un réseau «participent à égalité à une seule et même histoire » ${ }^{32}$, ou encore que tous ces éléments sont «capables dans une même mesure d'agir les uns sur les autres » ${ }^{33}$ ? Ces thèses ont certes le mérite de l'originalité apparente, sont certes compatibles avec la posture relationniste et sémiologique revendiquée par la théorie de l'acteur-réseau, mais elles impliquent l'abandon pur et simple des catégories traditionnelles de la sociologie. Enracinées dans une critique légitime du réductionnisme, elles débouchent sur un «jeu littéraire ${ }^{34}$ qui sacrifie bel et bien l'essentiel du projet scientifique de la sociologie des sciences.

\section{UN PROGRAMME DE RECHERCHE ACTIONNISTE EN SOCIOLOGIE DES SCIENCES}

L'examen critique de ces orientations montre que la définition d'un programme de recherche non réductionniste pour la sociologie des sciences n'est pas

29 M. Callon, Actor-Network Theory - The Market Test in Actor Network and After, Workshop Keele University, 1997

30 B. Latour, Les microbes. Guerre et paix, Paris, Pandore, 1984, p.42.

31 B. Latour, The politics of explanation: an alternative in S. Woolgar (ed), Knowledge and reflexivity, London, Sage publications, 1988, p. 173.

32 M. Callon (ed), La science et ses réseaux. Genèse et circulation des faits scientifiques, Paris, La découverte, 1989, p. 113.

33 J. Law, Traduction/Trahison - Notes on ANT, Lancaster University, 1997

34 Pour des illustrations de ce jeu, cf. M. Callon, La domestication des coquilles Saint-Jacques et des marins-pêcheurs dans la baie de Saint-Brieuc, in «L'Année sociologique », 36, 1986; B. Latour, Where are the missing masses? Sociology of a few mundane artifacts, in Bijker W., Law J. (eds), Constructing networks and systems, Cambridge, MIT Press, 1993. 
chose aisée. Il faut qu'il soit tout à la fois ambitieux - ouvert sur l'intégralité du processus de recherche — , méthodique - capable de définir avec précision la nature de son registre explicatif - et réaliste - conscient de ses limites et à même de s'enrichir de la pluralité des points de vue sur son objet d'étude. Je n'ai pas la prétention d'affirmer que seul un programme sociologique d'inspiration actionniste est en mesure d'occuper pleinement l'espace de contraintes simultanées ainsi défini. Mais je soutiens qu'un tel programme peut y prendre une place de choix dès lors qu'il lui est donné une formulation particulière. Cette formulation porte sur trois points essentiels: une conception forte de l'acteur social, un abandon du causalisme pour un rationalisme élargi non exclusiviste, une réinterprétation du principe de symétrie. J'essaierai d'illustrer brièvement ces points à partir d'une étude empirique menée sur le développement du secteur de recherches biomédicales consacrées aux maladies à 《prions ${ }^{35}$.

\section{Le primat de l'acteur scientifique}

Quelle conception de l'acteur doit faire valoir un programme de recherche actionniste en sociologie des sciences? A l'évidence une conception forte qui se différencie nettement de celles inhérentes au programme fort comme à la théorie de l'acteur-réseau. Le primat de l'acteur porte sur deux niveaux complémentaires:

A un niveau empirique élémentaire, cela passe par l'observation minutieuse des dimensions à la fois intentionnelles et significatives à l'œuvre dans l'exercice quotidien de la pratique scientifique. Le sociologue n'a pas à partir de la croyance a priori en l'indissociabilité de la nature et de la société, ni même du caractère indéterminé de la nature. Il part de la réalité des intentions, des motivations, des structures de préférences telle qu'elle se donne à voir à travers la pratique et le discours des acteurs scientifiques.

A un niveau de généralisation plus élevée - celui qui a été largement ignoré en sociologie des sciences dans la mesure où elle a été longtemps dominée par une perspective hyperempiriste - , cela passe par la typification de ces pratiques et discours et l'élaboration de logiques comportementales simplifiées à partir desquelles il devient possible de reconstruire les raisons suffisantes, au niveau microsociologique, de phénomènes macrosociologiques à expliquer.

Illustrons chacun de ces points à partir d'un exemple limité. Qu'observe-t-on lorsque l'on s'attache à reconstituer la transformation d'un domaine d'étude biomédical tel que celui des maladies à prions sur près de vingt années? Un groupe tout d'abord très restreint d'individus qui interagissent, développent des stratégies de recherche, élaborent des dispositifs expérimentaux, publient des articles et des rapports de recherche, s'insèrent dans des lignes de coopération internationale, entrent en contact avec les représentants de sphère institutionnelle très diverses, etc. bref, des individus engagés dans les divers aspects de la vie scientifique. La tâche du sociologue actionniste n'est pas de faire de tous ces éléments une strate épiphénoménale réductible à un niveau conceptuel exogène pour reprendre une catégorie du programme fort, ni même d'affirmer l'unité de ces éléments en pos-

35 M. Dubois, La communauté scientifique française des maladies à prions : évolutions démographique et stratégique, groupe de recherche Sociologie de la science, communication pour le congrès de l'AISLF, 2000. 
tulant l'existence d'un réseau dans lequel tous les éléments seraient interchangeables et où le sens ne cesserait d'évoluer. Son objectif est de donner à comprendre les catégories, les principes, les préférences propres à ces acteurs et de montrer de quelle manière ces éléments peuvent être des forces agissantes du point de vue du domaine de recherche considéré comme des relations entre ce domaine et son environnement direct ou indirect. Bref montrer comment non pas un paysage intellectuel, institutionnel et organisationnel préexistant détermine $a$ priori des actions scientifiques, mais comment ces actions participent de la production de ces paysages.

A un niveau différent - celui de la généralisation précédemment évoqué —, et tout en conservant le même exemple, on voit bien qu'à partir du moment où les chercheurs investissent collectivement le domaine des maladies à prions, il est possible de commencer à raisonner sur des données agrégées. Le début des années quatre-vingt-dix est marqué par un double phénomène: une accélération du recrutement des chercheurs et surtout une stabilisation des entrants. Le schéma dominant n'est plus celui de l'entrée furtive. Les chercheurs entrent et maintiennent leur intérêt en publiant régulièrement. Ce qui s'explique notamment par le fait qu'à partir de cette période des fonds européens puis nationaux ont été investis d'abord progressivement puis massivement pour soutenir la recherche. Dès lors qu'il dispose de ces données, le sociologue actionniste est en mesure de prendre du recul par rapport à l'observation empirique élémentaire en reconstruisant derrière la mobilité de l'intérêt scientifique des logiques sociales fondées sur la typification des comportements individuels. La transformation collective d'un domaine de recherche complexe devient compréhensible à partir d'un travail de formalisation de raisons strictement individuelles.

\section{Un rationalisme «élargi» non exclusiviste}

Cette référence à la nature compréhensive de la démarche sociologique me conduit naturellement vers le second principe général de l'actionnisme tel qu'il doit être à mon sens appliqué en sociologie des sciences : l'abandon du causalisme pour un «rationalisme élargi non exclusiviste».

Reprenons notre illustration. Il existe globalement trois hypothèses sur l'origine des maladies à prions: les hypothèses virologiques, les hypothèses protéiques et les hypothèses dites mixtes. L'hypothèse aujourd'hui dominante dans la communauté scientifique internationale est la seconde - c'est celle qui a valu à $S$. Prusiner son prix Nobel. Mais comme bien souvent le consensus autour de cette hypothèse n'est pas parfait. Un grand nombre de chercheurs français notamment continuent à privilégier les hypothèses virologique ou mixte. Lorsque l'on interroge par exemple J.-Ph. Deslys sur sa stratégie de recherche à long terme il fait observer qu'il a eu très tôt le sentiment que «certaines données ne cadraient pas parfaitement avec l'hypothèse du prion seul». D'où l'idée de développer sur plusieurs années des manipulations pour étudier les accumulations de PrP anormales. Dans la même perspective, D. Dormont et Lazmézas soulignent que si les arguments avancés par les tenants de l'hypothèse prion seule semblent convaincants il est tout aussi possible de produire des contre arguments point par point. «Si l'hypothèse la plus populaire et la plus originale demeure celle d'une protéine infectieuse, écrivent-ils, elle ne répond pas facilement à toutes les questions. Une 
somme de preuves partielles ne constitue pas une preuve absolue. Il existe donc encore de la place pour un agent infectieux associé à la PrP anormale».

De quelle manière rendre compte de ce comportement des chercheurs français dans cette controverse non encore fermée sur l'origine des maladies à prions? Il est possible de partir d'un constat d'indétermination rationnelle et d'interpréter la réticence des chercheurs à l'égard de l'hypothèse protéique comme une conséquence de leur conformisme intellectuel. Les chercheurs français ont très souvent été formés dans la tradition de la virologie, cette tradition influerait mécaniquement comme un cadre de pensée, une idéologie professionnelle qui les empêcheraient de concevoir le bien fondé des arguments de leurs adversaires. Il est également possible d'imaginer une variante matérialiste de ce causalisme dans laquelle cet attachement irrationnel à une hypothèse apparemment réfutée par les faits ne serait finalement rien d'autre que la conséquence de la volonté inavouable d'une corporation professionnelle de sauvegarder à tout prix un certain nombre de biens symboliques et matériels.

Abandonner le causalisme, c'est abandonner ce type d'explication - ou du moins c'est ne recourir à ce type d'explication par le conformisme ou l'intérêt matériel qu'en dernière instance. L'approche actionniste des sciences fait de l'étude des «raisons» qu'ont les acteurs de préférer une théorie à une autre, une hypothèse à une autre, un de ses axes centraux. Mais ces raisons sont distinctes de celles décrites par la tradition épistémologique. Elles ne s'opposent pas à la théorie de la rationalité à laquelle L. Laudan identifie la philosophie des sciences, mais viennent en quelque sorte l'étendre ou la compléter sur la base d'une théorie de la rationalité élargie. Ces raisons, ou «bonnes raisons » pour reprendre la terminologie proposée par R. Boudon ${ }^{36}$, n'ont pas de caractère strictement contraignant et tiennent leur valeur persuasive pour partie des caractéristiques de la situation dans laquelle elles sont produites ou mobilisées. Une tâche essentielle de l'analyse actionniste des sciences consistera à reconstruire les conditions de crédibilité des arguments échangés par les scientifiques, et partant à identifier dans une perspective sociologique la dynamique argumentative propre à son domaine d'étude.

\section{Réinterpréter la symétrie}

Ce travail est indépendant de la valeur de vérité des croyances, hypothèses ou théories analysées. Il n'est pas limité dans son application par un critère de démarcation de type épistémique. Dans sa discussion de l'étude de cas proposée par H. Collins, A. Franklin montre bien que le consensus scientifique autour d'une théorie n'émerge pas toujours sur la base de raisons contraignantes. Il ne propose pas une caractérisation sociale du système de raisons «fortes » qu'il identifie, mais on ne peut lui reprocher de ne pas avoir réaliser le travail qui nous revient en tant que sociologue. Ceci me conduit au dernier point permettant de définir ici de façon au moins programmatique l'approche actionniste des sciences: la nécessaire réinterprétation du principe de symétrie.

Il faut à mon sens non pas renoncer au principe de symétrie comme nous y invitent parfois les épistémologues mais le réinterpréter pour l'intégrer dans l'ensemble des conditions générales permettant d'apporter une explication solide des

36 R. Boudon, L'art de se persuader des idées douteuses, fragiles ou fausses, Fayard, Paris, 1990. 
croyances collectives. Le principe de symétrie pose comme condition préalable à la recherche la suspension de toute évaluation quant à la validité potentielle de son objet. Il représente un impératif de neutralité mais, et ce point mérite d'être souligné, il ne dit rien quant à la nature des «facteurs» à prendre en compte. Ce principe par ailleurs s'apparente à une exigence de méthode: c'est un point de départ que se donne le sociologue, mais pas nécessairement un point d'arrivée.

La disqualification relative de ce principe s'explique par l'usage particulier qui en a été fait: il a servi de caution à la tentative de rendre compte de la nature du processus de recherche en ayant recours de façon à la fois systématique et plus ou moins voilée à des catégories explicatives jusqu'alors réservées à l'explication des croyances fausses. Lié à une conception causaliste de la production des croyances scientifiques, il a représenté au final pour les représentants du programme fort un objectif à atteindre plutôt qu'une étape première ou temporaire. Ces remarques nous indiquent la voie à suivre pour le réorienter conformément aux exigences générales d'une approche actionniste des sciences. Il est nécessaire de réaffirmer sa nature méthodologique et de l'associer à la théorie de la rationalité élargie précédemment évoquée. L'analyse actionniste des sciences pourra être dite symétrique dans la mesure où elle se propose d'identifier derrière l'adhésion des acteurs scientifiques à une hypothèse vraie ou une hypothèse fausse, une théorie dominante ou marginale, un même ensemble de « raisons »-ensemble distinct de celui étudié par l'épistémologie. Interprété de la sorte, ce principe de symétrie permet de maintenir l'ouverture du domaine d'investigation de la sociologie des sciences tout en l'affranchissant des dérives réductionnistes qui par le passé ont davantage contribué à discréditer qu'à établir la légitimité de son point de vue.

CNRS - GEMAS

Maison des sciences de l' homme, Paris 